Ambiances

anbiances Environnement sensible, architecture et espace urbain

3 | 2017

Animer l'espace public ? Entre programmation urbaine et activation citoyenne

\title{
Danse, la cité. Infiltration chorégraphique d'une place publique
}

Danse, la cité. Choreographic infiltration of a public place

\section{Léna Massiani}

\section{OpenEdition}

\section{Journals}

Édition électronique

URL : http://journals.openedition.org/ambiances/1022

DOI : 10.4000/ambiances.1022

ISSN : 2266-839X

Éditeur :

Direction Générale des Patrimoines - DAPA - MCC, UMR 1563 - Ambiances Architectures Urbanités (AAU)

\section{Référence électronique}

Léna Massiani, «Danse, la cité. Infiltration chorégraphique d'une place publique », Ambiances [En ligne], 3 | 2017, mis en ligne le 10 décembre 2017, consulté le 20 avril 2019. URL : http:// journals.openedition.org/ambiances/1022 ; DOI : 10.4000/ambiances.1022

Ce document a été généré automatiquement le 20 avril 2019

Ambiances is licensed under a Creative Commons Attribution-NonCommercial-NoDerivatives 4.0 International License. 


\title{
Danse, la cité. Infiltration chorégraphique d'une place publique
}

\author{
Danse, la cité. Choreographic infiltration of a public place
}

\author{
Léna Massiani
}

\section{Introduction}

1 La nécessité d'engager une réflexion sur la participation collaborative du spectateur dans une création de danse in situ a débuté par la mise à disposition corporelle des intentions chorégraphiques qu'il m'a fallu développer lorsque celles-ci ont été déplacées en dehors de leur milieu habituel. Plonger l'écriture chorégraphique ailleurs que dans un théâtre n'est pas une chose anodine. Cela relève d'une détermination particulière consistant à l'ancrer dans les circonstances d'un lieu faisant lui-même partie d'une réalité.

«Une réalité que l'artiste veut faire plus que représenter, ce qui l'amène à délaisser les formes classiques de représentation pour leur préférer la mise en rapport directe et sans intermédiaire de l'œuvre et du réel. Pour l'artiste, il s'agit bien de tisser avec le monde qui l'entoure, de même que les contextes tissent et retissent avec la réalité. » (Ardenne, 2002, p. 18)

Selon le modèle de l'art contextuel décrit par Paul Ardenne, le geste artistique, performatif, chorégraphique devient emblématique d'un désir d'implication, d'une action relevant d'une expérimentation de la ville puisqu'il interroge notre rapport à la réalité urbaine. Autrement dit, l'espace urbain porte le discours, oriente et façonne l'action de l'expression esthétique. Il permet d'insister sur le lien politique et social que l'engagement artistique vient renouer. Il porte l'expérimentation sur les lieux des pratiques quotidiennes et amène le public au-delà de sa posture de spectateur. Il le place ainsi face à des artistes cherchant l'interaction avec « des individus participant à la sphère publique. » (Fraser, 1999, p.17). En son cœur, les habitants font partie des sujets. Et 
selon Marie Fraser, puisqu'ils en font partie, la relation qui se joue, tout comme les espaces investis, doivent faire l'objet d'une attention particulière.

3 Pour l'artiste, investir l'espace urbain peut engendrer un désir d'adresse différent. Plusieurs possibilités s'offrent à lui : parcours, déambulation, participation, infiltration. Dans tous les cas, il semble important de poser la question du rapport entre ce qu'offre un site et le dispositif choisi : est-il adapté à une déambulation? Où peut être placé le public ? Comment le garder volontairement à distance ? De quelle manière l'inviter à une participation?

4 Ma recherche doctorale engageait une réflexion sur le rapport au public en danse in situ (Massiani, 2011). En suivant le processus de Danse à tous les étages ${ }^{1}$, performance réalisée en appartement habité, l'enjeu était d'interroger et d'éprouver les conditions d'élaboration d'un rapport non frontal, en même temps que d'élaborer un dispositif permettant de confronter et de confondre les postures traditionnelles de regardant et de regardé. Or, au fil de la recherche, la question de la relation ne se posait plus en ces termes. Après avoir voulu assimiler la vie à l'art, après avoir voulu la présenter en s'emparant de son contexte réel, il semblerait que les artistes cherchent désormais à s'immiscer non seulement dans la vie mais aussi dans la vie sociale. Les pratiques artistiques participatives, que Louis Jacob (2007) invite à considérer comme une forme d'art public s'immergeant pleinement dans le territoire qu'elles investissent, prennent différentes formes. Entre sensibilisation à l'art et à la culture et éducation artistique, les pratiques participatives tentent selon l'auteur d'améliorer le cadre de vie dans un quartier. Leur enjeu principal concerne donc l'accompagnement des habitants. Il s'agit pour les artistes de leur offrir un espace d'écoute et d'expression, en valorisant leurs capacités, en leur permettant d'être un acteur de leur quartier, en les aidant parfois à formuler leurs besoins et leurs revendications. Pensée comme une action collective, l'on peut se demander de quelle façon intégrer la parole des habitants sur le terrain de la création ; de quelle façon impliquer leurs propositions? L'artiste devient-il un animateur ou un médiateur? Quelle relation s'invente alors?

5 C'est ainsi qu'à partir de ce raisonnement, et sur le principe méthodologique d'une recherche-création ${ }^{2}$, j'ai poursuivi la réflexion sur la relation au public en passant de l'intérieur à l'extérieur, de l'espace domestique à l'espace de la rue, d'un appartement habité à une place publique. Danse, la cité, projet chorégraphique de la recherche, a été pensé comme une action artistique urbaine s'inscrivant dans une démarche de (co)création. Des habitants sont invités à interroger leur lien avec une place publique de leur quartier. Ils sont invités à réaliser un geste dansé et performé faisant état de ce lien. Il s'agit de rendre visible ce que ces habitants sensibilisés à l'écoute du chorégraphique sont en mesure de percevoir, d'expérimenter, d'évaluer pour imaginer, inventer et concevoir avec leur corps : quelles relations entretiennent-ils avec ce lieu de passage, de transition, d'arrêt et de rencontre, de rendez-vous et de manifestation, de célébration et de fête? De quelle façon la place publique peut-elle être traversée et donc façonnée par des trajets? De quelle manière s'y arrêtent-ils, y passent-ils ? Réduire la réflexion à ces questions reviendrait, me semble-t-il, à ignorer l'influence que peut avoir sur notre gestualité un espace architecturé. Avec ce projet, ce qui m'intéresse est d'expérimenter la manière dont nous nous laissons conduire par les places publiques que nous traversons: qu'est-ce qui nous est donné? Les places publiques sont-elles toujours propices à la rencontre, au rendez-vous, au jeu, aux rassemblements? 
6 Dans une démarche d'auto-poïétique ${ }^{3}$, le présent article a pour objectif de restituer l'expérience Danse, la cité dans l'ensemble de son parcours. Afin de mieux considérer la manière dont j'ai accompagné les habitants dans la découverte sensible de leur quartier, de mieux comprendre comment se sont révélées à eux les places publiques et ce qu'ils ont décidé d'y créer, je ne pouvais faire l'économie d'une description argumentée des différentes étapes de recherche qui ont constituées le projet dans son ensemble. Pour cela, nous suivrons les étapes des deux temps forts révélateurs du projet.

\section{Danse, la cité : deux temps forts, deux quartiers}

\section{Le Mail de l'îlot Bièvre ${ }^{4}$}

7 Souhaitant prolonger la thématique de l'exposition L'îlot Bièvre, le corps et l'espace ${ }^{5}$, la directrice du centre d'animation a lancé un appel à projet ouvrant la voie à d'autres disciplines qui interrogeaient la relation entre le corps et l'architecture. Ce contexte m'a paru particulièrement favorable au projet de recherche que je débutais. Le Mail de l'îlot Bièvre étant un terrain de création singulier au cœur d'un site d'habitat social, j'ai répondu à cet appel. La question posée au travers de l'exposition était la suivante : comment améliorer et ouvrir l'îlot Bièvre en prenant en compte le corps? Je l'ai transformée et j'ai proposé : comment, avec le corps, expérimenter l'îlot Bièvre en vue de le remodeler pour se l'approprier?

8 A l'occasion du vernissage de l'exposition, l'invitation à participation a été présentée aux habitants du quartier. Par la suite, pour les sensibiliser au projet et au travail qui y sera développé, le centre d'animation a effectué des rendez-vous réguliers avec les habitants, mais aussi avec leurs usagers ou encore avec le public du théâtre 13, voisin du centre. Des discussions avant et après des cours, des présentations d'extraits de projet de la compagnie, des affichages dans les commerces ont été réalisés dans ce but. Après quoi, un groupe de trois participants ${ }^{6}$ a rejoint le projet. Avec les deux danseuses ${ }^{7}$, l'équipe était donc constituée de cinq personnes.

\section{La place Louis Baillot ${ }^{8}$}

9 L'invitation de l'association Ab Ovo à participer au festival Goutte d'Or en fête organisé par la salle Saint-Bruno (10 et 13 juin 2015), consistait à donner la parole à l'habitant dans son quartier en investissant la place Louis Baillot. Ab Ovo produit en effet des œuvres participatives dans lesquelles l'accent est mis sur la sensibilisation et la médiation. Comme dans le $13^{\mathrm{e}}$ arrondissement, j'ai rencontré les habitants et les usagers de la salle Saint-Bruno à plusieurs reprises et au cours de différents événements : la préparation d'une soupe partagée au jardin la Goutte verte a permis par exemple de présenter le projet, ou encore le lancement des préparatifs du festival au centre Barbara m'a donné l'occasion de rencontrer différentes associations susceptibles de relayer l'information auprès de leur public. C'est ensuite sur le terrain que ces dernières ont sensibilisé les habitants grâce à des discussions avant et après une activité. Durant ce deuxième temps fort, l'équipe a compté deux participants ${ }^{9}$ et une danseuse ${ }^{10}$.

Les deux temps forts ont été abordés selon les mêmes étapes de travail. Un premier moment a consisté en la rencontre avec les participants et entre participants grâce à une sensibilisation au chorégraphique, de l'écoute de soi à la rencontre avec les autres. Afin 
de pouvoir accompagner de la manière la plus juste et la plus sensible les participants, mais également dans le but de pouvoir partager et échanger avec eux sur le fond comme dans la forme, lors d'une étape intermédiaire je suis partie à la découverte des deux sites. Ensuite, les habitants ont à leur tour observé activement les places sur lesquelles nous sommes intervenus. A la suite de quoi, nous pouvions partager nos expériences, et les participants définir des axes de recherche chorégraphique.

\section{Sensibiliser le corps des habitants au chorégraphique}

\section{Le chorégraphique}

L'expression qui est la mienne, la chorégraphie in situ, m'a obligée à considérer l'espace urbain sous l'angle du mouvement. Dépasser le cadre institué par le spectacle vivant m'a poussé à aller à la rencontre du public, pour finalement inventer avec lui dans l'espace urbain. Après m'être intéressée à la relation qu'entretiennent le corps et l'architecture, ma recherche chorégraphique s'est nourrie du rapport qui se noue avec l'environnement urbain. Ce qui m'importe est de révéler, par des actions dansées, des espaces que nous ne voyons pas en même temps que créer nos propres espaces d'action. Par sa simple (et non banale) présence, le corps en se logeant là où il n'est pas attendu, en soulignant une ligne, une forme, en utilisant un ensemble de détails - un coin, un recoin, une paroi, un contour, un sol, une matière, un mur - peut faire apparaître des volumes, des tensions, des directions, entre horizontales et verticales, des rythmes et des dynamiques : un espace sensible s'esquisse. De là, je positionne le corps du danseur en relation avec l'espace qu'il investit pour élaborer un geste qui prend sa source au cœur de l'urbain. Et donc, la relation qui se tisse repose sur un certain nombre d'expérimentations sensibles : prendre conscience des volumes du corps, de la place qu'il peut investir, de l'espace qu'il peut remplir, de ses contours et de ses limites.

12 S'immerger, sentir et s'imprégner, pour pouvoir se positionner, se situer, mettre en valeur des interstices, créer des perspectives, devenir des points mobiles, souligner, pointer, cette démarche m'a conduite à développer une écriture chorégraphique basée sur une recherche active que les danseurs peuvent avoir d'un espace urbain. Afin qu'ils puissent s'y engager, en avoir une connaissance fine s'est révélé nécessaire. De ce point de vue, le corps dansant, sensible, est devenu un moyen de partir à la découverte d'un site, de comprendre l'environnement urbain dans lequel s'inscrivait le geste et de le qualifier en regard des sens qu'il met en éveil. Nous le voyons, grâce aux propriétés du chorégraphique, le corps dansant développe certaines compétences qui peuvent faire de lui un outil atypique, considérant « le sentir » comme moyen fondamental d'expertise. Avec Danse, la cité, ce sont ces propriétés qu'il m'intéressait de développer avec le corps des habitants, un corps agissant pouvant ouvrir sur une incroyable capacité à saisir des comportements kinesthésiques en fonction d'un espace urbain. Mais quelles sont ces propriétés? De la quotidienneté à l'approche phénoménologique du corps, de l'état de corps à la performativité, de la théâtralité à l'orchésalité, je catégorise le chorégraphique selon quatre propriétés qui me donnent la possibilité de ne garder que les caractéristiques proprioceptives de l'expérience sensible que développe le corps dansant : 1. expertise proprioceptive du pré-mouvement; 2. écoute de l'infra-sensible: le corps comme capteur(s) sensoriel(s) ; 3. entrecroisement des qualités de l'effort labaniennes (Poids, Espace, Temps, Flux): de l'intérieur du corps à l'environnement urbain; 4. 
dialogue entre la mise en éveil corporelle et l'identité spatiale : de la kinésphère ${ }^{11}$ au sens multidirectionnel (ou la découverte de multiple dimension à partir du corps d'une part et, en fonction d'un espace donné, d'autre part).

\section{Illustration 1 : La kinésphère schématisée par l'Icosaédre}

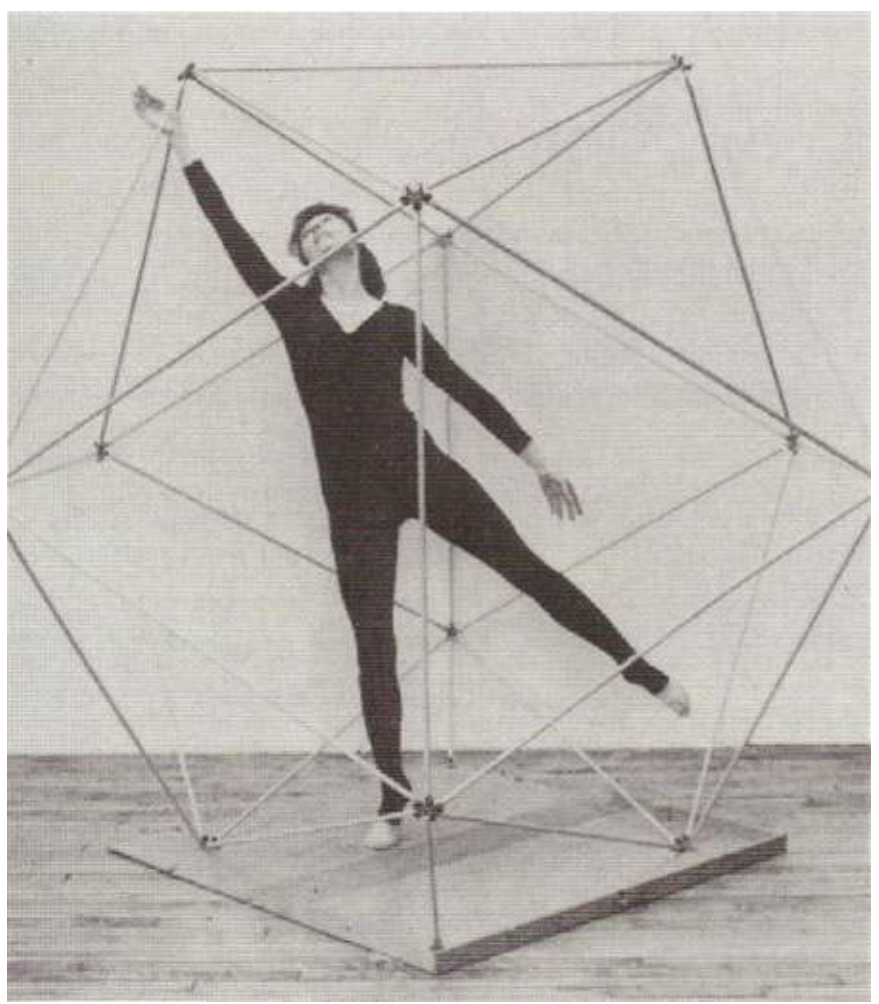

Source et copyright: Schwartz, Elisabeth. 2000. Les Trames architecturales du mouvement chez Rudolf Laban. Nouvelles de Danse. $n^{\circ}$ 42/43, p. 44.

\section{Mise en corps des habitants}

Pour aborder le rapport entre le corps et l'environnement urbain, il me semblait important et nécessaire de proposer aux habitants une première étape consistant en une phase de prise de conscience des espaces internes du corps. Cette étape ouvrant au sensible et permettant l'éveil des capteurs sensoriels du corps s'apparente aux techniques somatiques (et en particulier celles des releases techniques). Il m'importait par là d'aboutir à une prise de conscience des mobilités internes et des micromouvements qui les accompagnent en vue de considérer la respiration comme une matière dense à partir de laquelle il devient possible de sentir les volumes du corps, ses points d'appuis, son poids et sa gravité, ses espaces charnières et de passage.

Portée à la verticale, j'ai associé cette approche du corps aux recherches du danseur et théoricien allemand Rudolf Laban (1876-1958). Celles-ci sont pertinentes à deux endroits. D'abord elles concrétisent l'idée qu'à partir du corps et de son intériorité, des mouvements peuvent être projetés et des espaces d'action créés. Ensuite, le chercheur a montré que nos actions quotidiennes sont pourvues de qualité qui diffèrent selon la manière dont elles sont projetées. En partant de ce postulat, nous pouvons définir 
l'espace que génère le mouvement humain que le chercheur conçoit comme une architecture vivante.

15 A partir de la kinésphère de chacun des participants a été appréhendée la relation entre l'espace et le corps en déplacement. Les participants ont décomposé les possibilités de mouvement selon des lignes de direction qui passaient toutes par le centre de la kinésphère, c'est-à-dire leur corps. Plusieurs trajets entre différents points ont été testés grâce à des lignes de direction (haut, bas, milieu, etc.). La proposition consistait en somme à expérimenter plusieurs combinaisons de mouvement projetées vers la kinésphére en se déplaçant d'un point $\mathrm{A}$ à un point $\mathrm{B}$ (haut, avec le coude/milieu avec le genoux/bas avec la tête, etc.).

Apparemment dépourvue de la perception sensible, cette conception du mouvement semblerait envisager le déplacement sous l'angle unique d'une construction géométrique de l'espace. Or, si cette façon de considérer la spatialité du corps permet de mieux évaluer notre rapport à ce qui nous entoure, la théorie de l'effort qui l'accompagne l'est tout autant. J'ai donc proposé aux participants de déterminer l'effort physique des mouvements possiblement projetés dans la kinésphère. D'après Laban (ibid.), toute activité, volontaire ou involontaire, nécessiterait et manifesterait un certain effort physique. Les mouvements, qu'ils soient conscients ou inconscients résulteraient de l'interaction entre quatre facteurs dynamiques : le poids, l'espace, le temps, le flux. Nous adopterions en effet, consciemment ou non, une façon d'être en mouvement qui se nuance entre deux attitudes extrêmes : s'abandonner au mouvement ou être contre lui. Le " poids » d'une action se ferait léger ou lourd, son « temps " pourrait être continu ou discontinu, son « espace » indirect ou direct, enfin son «flux » serait libre ou contrôlé. Quelques combinaisons ont été pratiquées. A partir d'actions comme lancer, tomber, sauter, repousser, les participants ont évalué en mouvement et de manière effective le poids, l'espace, le temps et le flux de celles-ci.

Le corps éveillé, le corps réveillé, la deuxième étape s'est appuyée sur cette écoute sensible et proprioceptive pour observer et découvrir les environnements urbains des deux temps forts de Danse, la cité.

\section{Observation sensible}

L'observation sensible s'est découpée en deux moments distincts. J'ai d'abord réalisé seule cette observation particulière en utilisant tout le potentiel que l'usage du pré-mouvement recèle. Sensibilisé au perceptif, à l'écoute de soi et à l'écoute des autres, se sont ensuite les habitants, qui dans un état de corps ordinaire sont venus observer le Mail de l'îlot Bièvre pour le premier temps fort et la place Louis Baillot pour le deuxième.

\section{Le pré-mouvement}

19 Aborder le Mail de l'îlot Bièvre et la place Louis Baillot par le pré-mouvement a permis de prendre en compte les atouts intuitifs d'une analyse fine du mouvement. Pour ce faire, je me suis écoutée, j'ai écouté les intentions de mes mouvements, j'ai écouté ce que l'on pourrait nommer la « micro-perception » qu'un état de corps debout est déjà en mesure de signifier.

« La posture érigée, au-delà du problème mécanique de la locomotion, contient déjà des éléments psychologiques, expressifs, avant même toute intentionnalité de 
mouvement ou d'expression. Le rapport au poids, c'est-à-dire à la gravité, contient

déjà une humeur, un projet sur le monde. » (Godard, 1998, p. 224)

20 Hubert Godard (1998) qualifie de pré-mouvement cet état de corps au monde qui influence notre organisation gravitaire et qui agit « sur la façon dont le sujet organise sa posture pour se tenir debout et répondre à la loi de la pesanteur dans cette position » ( Ibid). Le pré-mouvement est une forme d'anticipation sur le mouvement. L'auteur décrit l'exemple de l'action de tendre un bras devant soi. Ici, le premier muscle à entrer en action est le muscle du mollet. Celui-ci, précise Godard, anticipe la déstabilisation « que va provoquer le poids du bras vers l'avant » (Ibid). Considérant l'espace dans lequel peut être porté une action corporelle, celle-ci s'articule indéniablement avec ce qui est présent autour. C'est, à mon sens, l'environnement dans lequel le corps est en présence qui influence et détermine ses pré-mouvements. Hubert Godard ajoute d'ailleurs à ce propos :

«L'architecture, l'urbanisme, les visions de l'espace, le milieu dans lequel évolue le sujet vont également avoir une influence déterminante sur son comportement gestuel. » (Ibid., p. 226)

Et ce comportement gestuel est pour ma part une source d'information considérable quant à la qualité d'un espace urbain.

M'intéresser au pré-mouvement m'a également permis de découvrir les amplitudes du corps; une amplitude qui va de l'imperceptible à l'agir. La respiration a alors été considérée comme un mouvement d'où émerge une multitude de capteurs sur le monde pouvant atteindre une connaissance des environnements urbains que nous traversons. Le socle de notre présence au monde est inscrit en nous, c'est une respiration, une préaction. La manière dont j'ai ensuite envisagé le pas qui a foulé les deux places publiques a été la conséquence de la rencontre entre mon espace interne et l'environnement autour.

\section{Le pré-mouvement, sur le terrain}

Sous ses aspects instinctifs, le principe de cette observation ne s'est pas actionné n'importe comment. L'intuition sous-jacente s'est organisée en plusieurs moments d'attention. Lorsque j'ai découvert les lieux, j'ai souhaité agir de la manière la plus neutre possible. Traverser l'espace, m'installer, m'assoir, regarder, lire, écouter. Je voulais avoir une impression de l'allure générale de ces places. Ensuite, en marchant spontanément, je me suis laissée porter et je me suis positionnée à différents endroits, dix pour le Mail, cinq pour Baillot. Le chemin entre les points était tout à fait intéressant à remarquer, puisqu'il résultait du pré-mouvement par lequel s'engageait ma marche. Il était donc signifiant de l'impulsion donnée par les lieux. En même temps, pour bien comprendre la relation du corps à l'espace, chaque station d'arrêt a fait l'objet d'un temps d'écoute : d'où part cette première sensation et vers quoi me porte-t-elle? A ce stade, je n'ai pas interprété le mouvement ou les déplacements puisqu'il s'agissait de comprendre ces deux places pour ce qu'elles sont et d'en saisir leur identité propre.

Dans un deuxième temps, mon regard s'est ouvert au monde et j'ai observé la composition de l'endroit, ses matières, ses sons, ses odeurs, mais aussi ses formes et la manière dont il se structure. Sur le Mail et à Baillot, j'ai observé les alentours, la disposition des commerces, des bars, ou encore la structuration des transports. Il était également important de remarquer si les espaces disposaient de végétation et de quel type; ou encore s'il y avait du mobilier urbain, à quel endroit, la manière dont il était agencé et pour quel usage. Tout ceci pour mieux saisir l'incidence que cela pouvait avoir 
sur les personnes qui font vivre les deux endroits. Et justement, la compréhension du mouvement constituant une part majeure de l'observation, je me suis intéressée à ceux des passants, mais aussi à mon propre mouvement. J'ai attaché une importance particulière à son déploiement et aux corps qui circulaient. Je me suis déplacée à l'intérieur, j'ai tracé, bougé en me demandant si le mouvement faisait partie de leur composition, de leur forme ou s'il était un ajout. Ce dernier point a éminemment été important. Nos déplacements pouvant être perçus comme une substance façonnant la ville, la place publique peut être entendue comme le noyau d'où ils rayonnent : est-ce que se sont les déplacements qui structurent son organisation ou son organisation spatiale qui influence les circulations?

\section{Approche proprioceptive du mouvement : l'observation des habitants}

A partir de l'observation réalisée grâce au pré-mouvement, celle faite par les habitants s'est effectuée en cinq étapes. Chacune a fait l'objet d'une approche individuelle, comprenant un temps d'écriture :

1. Déambuler en étant attentif au poids, à l'espace, au temps, au flux des déplacements. Remarquer les temps d'arrêt, la vitesse de marche; une accélération, une décélération: qu'est-ce qui influence ou nourrit un déplacement? Quelle relation est-il possible de remarquer avec l'agencement de la place? Est-ce par hasard qu'un mouvement est effectué?

2. Observation générale de l'espace qui nous entoure : en se déplaçant sur la place, être attentif à la manière dont est agencé l'espace : son dessin, sa forme, sa géométrie ; les horizontales, les verticales, les vides, les pleins, les interstices. Puis situer la signalétique, est-elle lisible, visible? Où se trouvent le mobilier et la végétation: quel type? quels usages? De quelle manière l'ensemble est-il structuré ?

3. Observer la place à partir des sens : être attentif aux matières, aux sons, aux odeurs, aux goûts, à ce qui est vu : qu'est-ce qui est touché, entendu, goûté ? De quelle façon? Est-ce que cela s'effectue dans l'immobilité, en déplacement, assis et combien de temps?

4. Observer par la motricité et l'action : tester différents points de vue. Associer au moins dix verbes d'action, comme par exemple "se cacher, courir, sauter, plonger, se coucher... ", à différentes qualités de mouvement parmi les suivantes: lourdement, légèrement, directement, indirectement, en continu/discontinu, libre, contrôlé. Qu'est-ce que cela change, comment et à quel endroit ?

5. Observer les déplacements des autres. Porter l'attention sur les chemins empruntés; observer les postures quotidiennes des passants, leurs habitudes, leurs attitudes, leurs temps d'arrêt ? Les emprunter. Y-a-t-il de la rencontre, du jeu?

\section{Mise en commun et choix des thèmes chorégraphiques par les habitants}

Suite aux observations, nous avons échangé et mis en commun nos impressions, nos sensations, nos réactions, nos remarques. Les observateurs ont exprimé leur parcours, le tout étant soigneusement noté sur un tableau dans plusieurs colonnes. A force de découpage et d'assemblage, nous avons pu découvrir « un » Mail de l'îlot Bièvre et « une » place Louis Baillot ${ }^{12}$. 


\section{Le Mail de l'îlot Bièvre} Glacière. Le Mail de l'îlot Bièvre se situe en son cœur, enclavé entre des tours d'immeuble. Il faut entrer à l'intérieur pour arriver en surplomb. Ensuite, nous devons descendre des escaliers pour finalement nous assoir devant le théâtre 13. Nous avons alors vu deux autres possibilités d'y entrer, un jardin et un autre escalier. Nous les emprunterons plus tard pour expérimenter une autre façon d'arriver sur le Mail. Nous avons écouté, goûté, et enfin nous nous sommes déplacés. La légèreté des déplacements n'a pas été ce que nous avons retenu. Au contraire. Nous étions invités à porter notre regard vers le haut, alors que l'intention du pas s'ancrait davantage dans le sol. Le fait d'être très bas par rapport au niveau des immeubles et de celui de la rue, et l'impression d'être à l'intérieur d'un espace bien qu'à l'extérieur, nous ont certainement incité à entrer encore davantage dans cet espace. Lors des déplacements plus spontanés, nous nous sommes laissés porter aux coins du carré de verdure qui se trouve au centre du Mail.

Cet espace, le carré, est très calme, minéral, et en son centre, une pelouse recouvre une ancienne fontaine devenue un espace de promenades canines. La bordure de cette ancienne fontaine fait également office d'assise. Ce site n'est pas une place publique comme les autres. Il donne d'abord l'impression d'être une sorte de cour intérieure entre des immeubles, destinée aux jeux des enfants et aux déambulations des chiens en laisse. Pourtant, cet espace n'est pas seulement connu de ses habitants proches. Il s'ouvre à un public des quartiers alentours, travailleurs et habitants, usagers du centre d'animation et spectateurs du théâtre 13. Les temps d'arrêts y sont cependant d'une courte durée. Entre pause-déjeuner et attente d'un spectacle, certains se laissent aller au temps d'une sieste. Essentiellement lieu de passage, en fin de journée, l'espace est traversé par de nombreuses personnes se rendant au supermarché bordant le Mail. Plusieurs entrées découpent l'espace en différents niveaux, lîlot Bièvre dispose en effet de plusieurs étages. Celles-ci sont empruntées suivant les objectifs de chacun, puisque quatre rues entourent la cité Glacière. Les différences de volume qui le composent confirment l'impression d'un dialogue entre l'enfermement et l'immensité.

La brutalité était l'image première que nous avions de l'îlot Bièvre. L'aspect peut accueillant du lieu venant justifier ce sentiment d'étouffement et d'empressement. Cette impression première a pu confirmer la contradiction du Mail qui désormais devenait plus claire. En effet, après l'observation, nous avons distingué deux attitudes, celle des personnes habituées à y venir, c'est-à-dire les habitants et celle « étrangère » à l'endroit. Nous retrouvions dans l'attitude des habitués ce que nous-mêmes pouvions vivre : ce lieu est un lieu de rendez-vous, et les habitants du quartier s'y retrouvent. Différentes activités y sont d'ailleurs régulièrement organisées, soit par le centre d'animation, soit par le théâtre. Malgré tout, nous remarquons que le site n'est pas toujours adapté aux jeux des enfants, que ce carré de pelouse ne peut pas être vraiment utilisé, qu'il doit être nettoyé lorsqu'un événement est prévu et qu'il ne permet pas une circulation fluide puisqu'il coupe les trajets. Ainsi, les personnes de passage ne s'y arrêtent pas, puisqu'elles n'y sont pas invitées. Finalement, avec ce qui s'y trouve, seuls les habitants parviennent à en faire un lieu de vie. Le désir de transformer le Mail en un espace de rencontre dans lequel prendre le temps, de le transformer en une place propice à l'accueil, au partage et au jeu a alors émergé. Les habitants ont souhaité tracer et (re)dessiner des chemins. Ils 
ont voulu ouvrir d'autres formes de passage (au sens très concret comme dans un sens plus poétique) pour pouvoir marquer de leurs empreintes le site et faire en sorte qu'il puisse être identifié et identifiable. A cet effet, deux orientations ont été définies: la traversée et le passage ; le poids et le contrepoids.

\section{La place Louis Baillot}

Située en bordure de trois routes à la circulation intense, la place Louis Baillot se perd dans le paysage urbain. Pas très claire à identifier, nous sommes restés un certain temps assis sur un banc, adossés contre un arbre ou un lampadaire. La rencontre avec les passants-habitants a été immédiate. Les trois bancs dont dispose la place sont constamment occupés, les gens viennent s'y arrêter et discuter. En nous déplaçant, nous avons été portés davantage vers l'avant. Nous ne savions pas quelle direction prendre, du fait même de l'aspect chargé de la place. Un fort déséquilibre nous a poussé, entre légèreté et profondeur. Les déplacements sont en effet assez vifs. Cela est sans doute dû à l'étroitesse du lieu. Par ailleurs, il nous a été difficile de nous arrêter, ou plutôt de trouver une "fin» au périmètre de la place. C'est la limite du trottoir qui nous a stoppés. Le pourtour de la place Louis Baillot n'est pas évident à voir. D'autant que la frise multicolore de l'autre côté de la rue porte le regard, elle en fait partie.

31 La place Louis Baillot est un espace qui n'est pas facile à distinguer et à identifier. Coupée en deux par trois arbres, deux réverbères et une poubelle, un côté est propice à la discussion et l'autre est réservé aux passants qui vont et qui viennent entre deux arrivées de bus. Il s'y passe beaucoup de choses, à l'image du quartier de la Goutte d'Or. Les énergies et les dynamiques partent dans toutes les directions. C'est une place de passage, c'est une place où l'on jette ses poubelles, où l'on vient nourrir les très nombreux pigeons, où l'on vient prendre l'air et discuter, mais aussi où l'on court pour ne pas rater son bus. Et comme l'architecture n'est pas identifiable, comme ses verticales, ses horizontales, son dessin ne sont pas lisibles, cela rend d'autant plus compliquée son observation. Et puis, assez vite au cours de la déambulation, le mur de graffiti est une accroche visuelle, il donne une couleur, une matière, et une identité à cette place.

Sous son aspect brouillon et tout azimut, la place Louis Baillot est un haut-lieu de rendezvous. Certaines personnes du quartier s'y retrouvent et discutent. Pour autant nous n'avons jusqu'alors traversé cette place que pour prendre le bus. Trop loin du centre de la Goutte d'Or, aucune activité n'y est vraiment organisée. Or, il se trouve que la place Louis Baillot est le départ de la parade de la Goutte d'Or en fête. C'est une belle occasion de la montrer sous un autre aspect. Les regards seront ouverts à l'écoute. Nous devons dès lors en révéler le «charme » et montrer qu'il n'est pas si difficile d'en faire un lieu de rendezvous phare du quartier. Nous devons dépasser son périmètre car l'espace étant très réduit (trois bancs, aucune table), peu de personnes peuvent en réalité s'y arrêter. Les bars alentour ne pourraient-ils pas devenir des espaces de rendez-vous? Nous devons ouvrir notre champ visuel de la place et ouvrir la place à notre regard. Le besoin de pouvoir montrer tout l'espace, des plus petites choses aux plus grandes a trouvé sa résonnance avec l'envie de se faufiler comme de s'implanter, d'ouvrir, d'agrandir, de dépasser. Deux orientations chorégraphiques ont été décidées : un travail sur la mesure et un jeu avec les distances. 


\section{La création : poser un geste, la danse écrite par les habitants}

\section{Sur le Mail de l'îlot Bièvre}

Prenant l'allure d'atelier chorégraphique, la recherche gestuelle s'est organisée en deux temps suivant les deux orientations choisies : la traversée et le passage, et le poids et le contrepoids. Le premier temps a donc été consacré à la marche. De l'équilibre au déséquilibre en passant par le débordement, la déconstruction des dynamiques de marche a été conduite par des intentions de chute, d'apesanteur, de lenteur, de suspension, d'arrêt, d'accélération et de décélération. Cela a été initié non pas dans les chemins habituels mais tout autour du carré de verdure, espace central du Mail.

Illustration 2 : Exploration de la marche

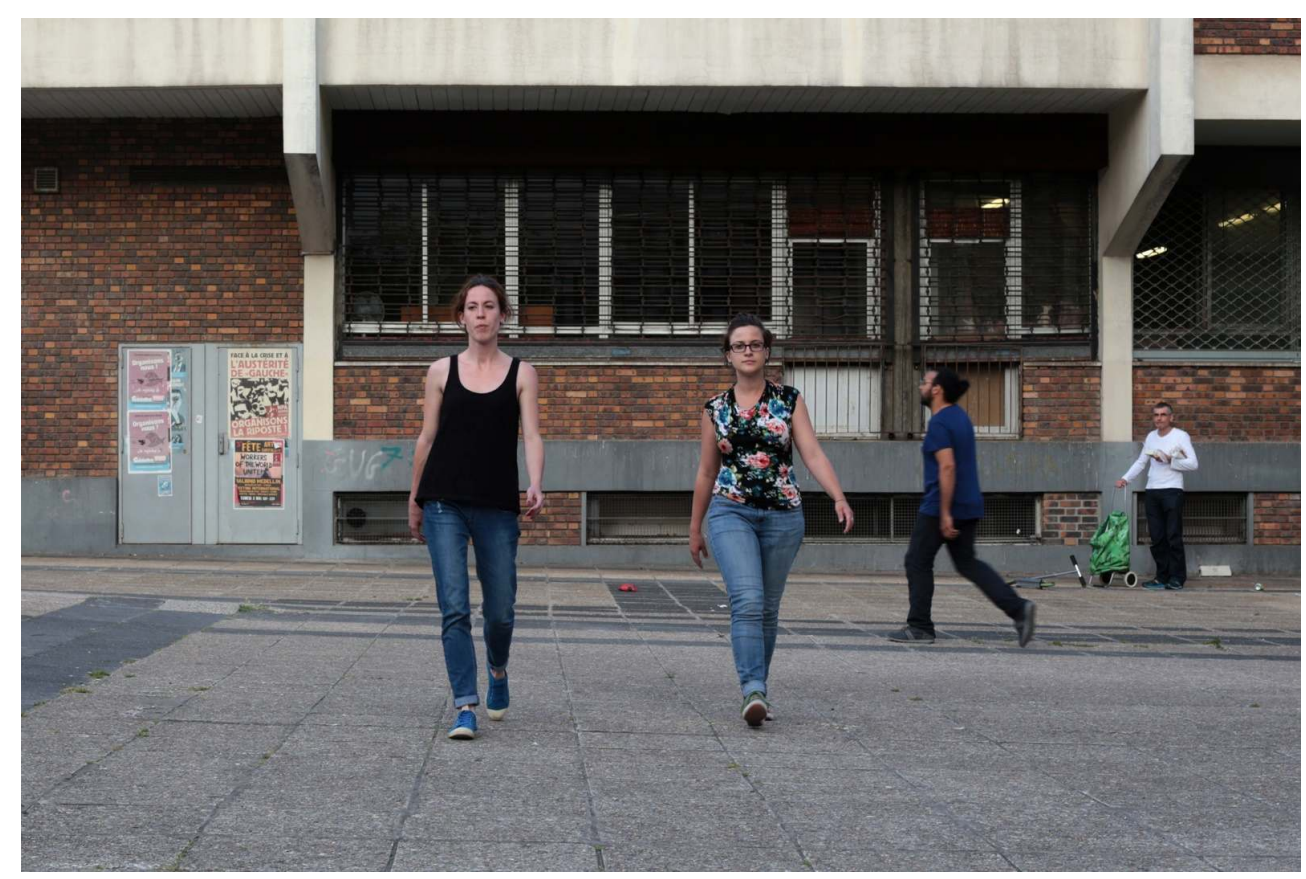

Source et copyright : Archive personnelle. (C) Julien Vincent

Le deuxième temps a fait l'objet d'un jeu plus direct de corps à corps avec l'architecture. Il ne s'agissait pas de se fondre dans la masse, mais plutôt de tenter de s'y accrocher selon différentes qualités de poids. Il était possible de la souligner avec le corps, mais surtout, les participants pouvaient être repoussés par elle, se repousser, être aspirés, chuter ou encore glisser sur les différentes surfaces du lieu. 
Illustration 3 : Exploration du corps à corps

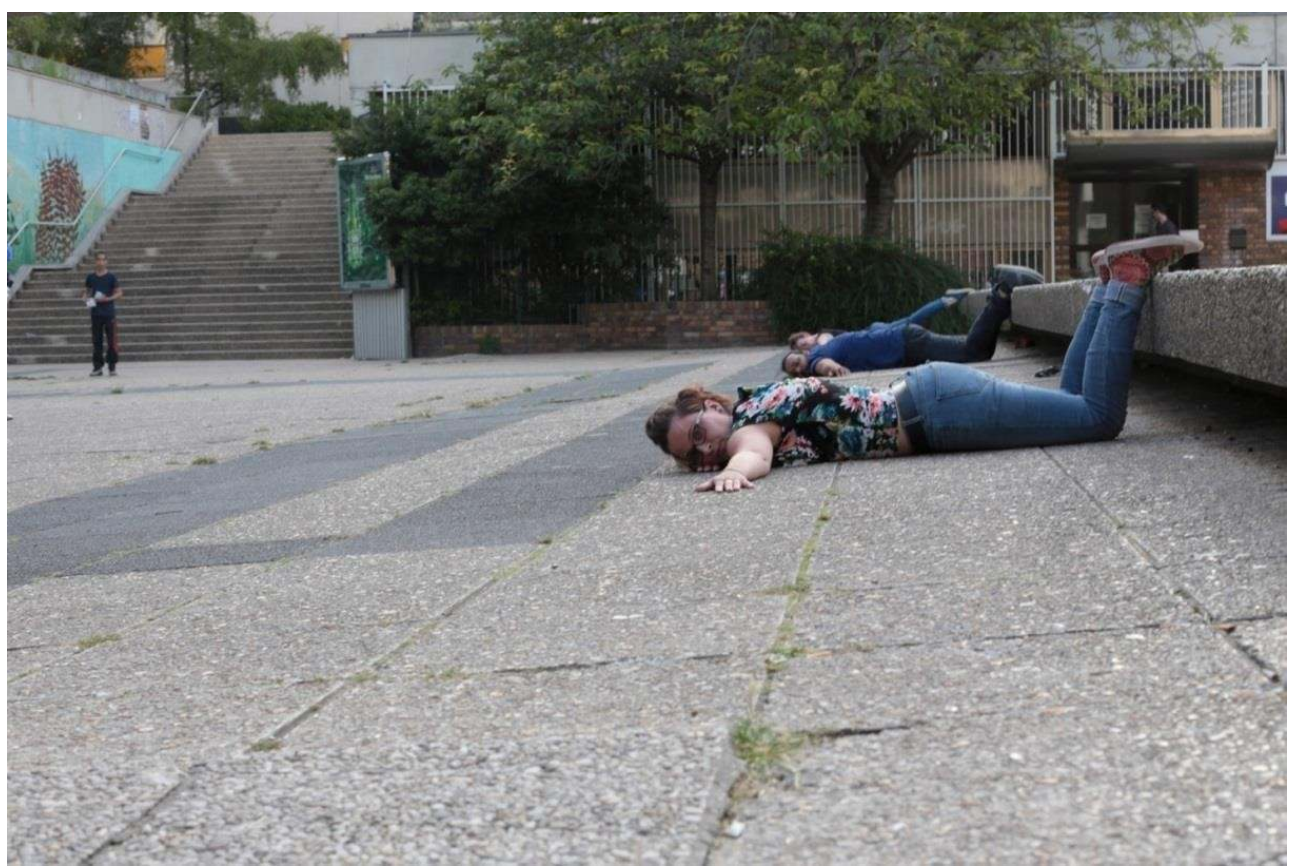

Source et copyright : Archive personnelle. () Julien Vincent

\section{Sur la place Louis Baillot}

L'exploration gestuelle a débuté par un jeu autour de la mesure (premier thème chorégraphique). Plusieurs pistes de recherche se sont révélées : qu'est-ce que le corps peut signifier d'un espace? Comment peut-il se situer et devenir un outil de mesure donnant à voir la place ? De quelle manière le corps peut-il la souligner, s'y imbriquer ou encore l'enrober? 


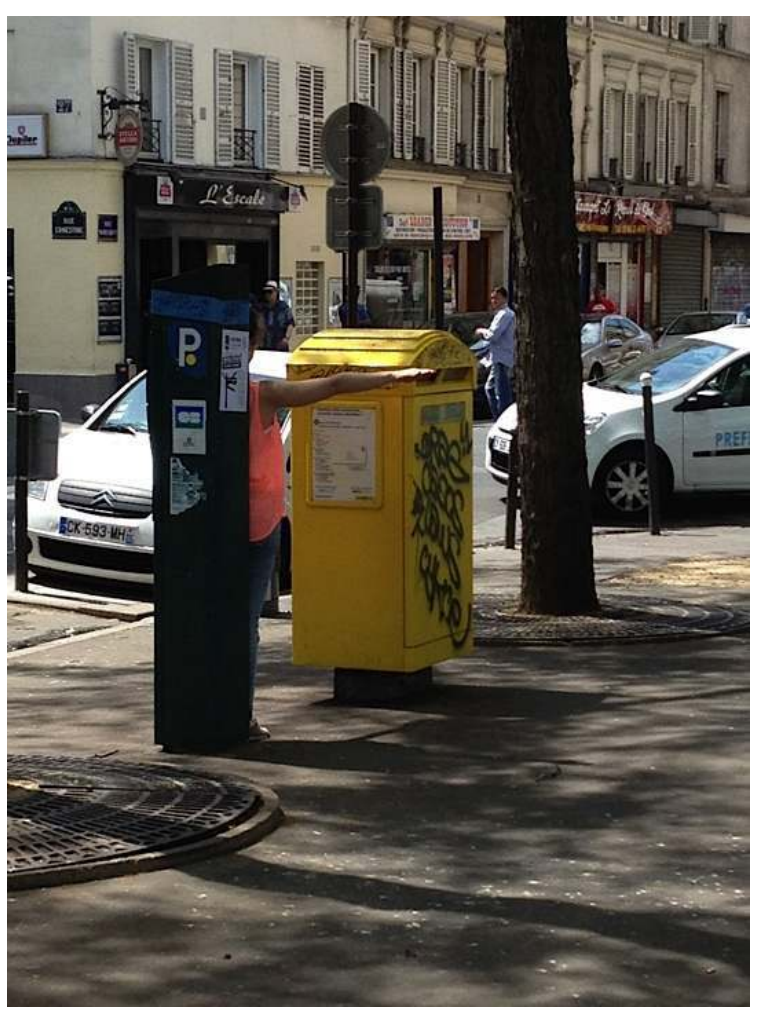

Source et copyright : Archive personnelle. @ Léna Massiani

Par la suite, le jeu avec les distances (deuxième thème chorégraphique) s'est appuyé sur l'idée que le corps peut être à la fois un point mobile et immobile de l'espace, tandis que la place Louis Baillot ne peut être que fixe: de quelle manière, en s'éloignant et en se rapprochant des différents points de l'espace, faire apparaître une distance et des volumes? Ces distances peuvent-elles devenir des espaces de passage? L'exploration a été complexifiée par l'envie de prendre en référence non plus uniquement l'espace autour, mais aussi les partenaires. Chacun est devenu une architecture avec laquelle il était possible d'interagir. Les participants ont trouvé des espaces de jeu improbables. Ils ont créé des surprises, des temps d'arrêt, des suspensions, des respirations que la place venait finalement porter.

\section{Réalisation}

Danse, la cité est une action artistique urbaine qui tente de changer « notre regard et notre écoute, la perception globale des formes et du temps urbain » (Augoyard, 2000, p. 21) et qui s'inscrit dans une démarche participative. Son dispositif est pensé sous la forme d'une infiltration de l'espace urbain. La performance s'insère ainsi par touche et par couche dans les espaces qu'elle investit sans qu'il n'y paraisse. Les participants se fondent dans un contexte et se rendant parfois peu perceptibles, pour reprendre les mots d'Augoyard. Présents dans l'espace de la place d'abord de manière quotidienne, ils amplifient ensuite une marche, passant de la mesure à la démesure, de l'équilibre au déséquilibre. Les participants se voient ensuite réaliser une danse dont le geste est inspiré des places. Sans début ni fin, pris dans le flux quotidien, l'infiltration pourrait se jouer en continue. Les 
applaudissements n'étant pas attendus, Danse, la cité commence de la manière dont elle s'est terminée et elle se termine de la manière dont elle a commencé.

\section{Sur le Mail de l'îlot Bièvre}

Ce média ne peut être affiché ici. Veuillez vous reporter à l'édition en ligne http://

\section{Sur la place Louis Baillot}

Illustration 5 : Danse, la cité / Place Louis Baillot

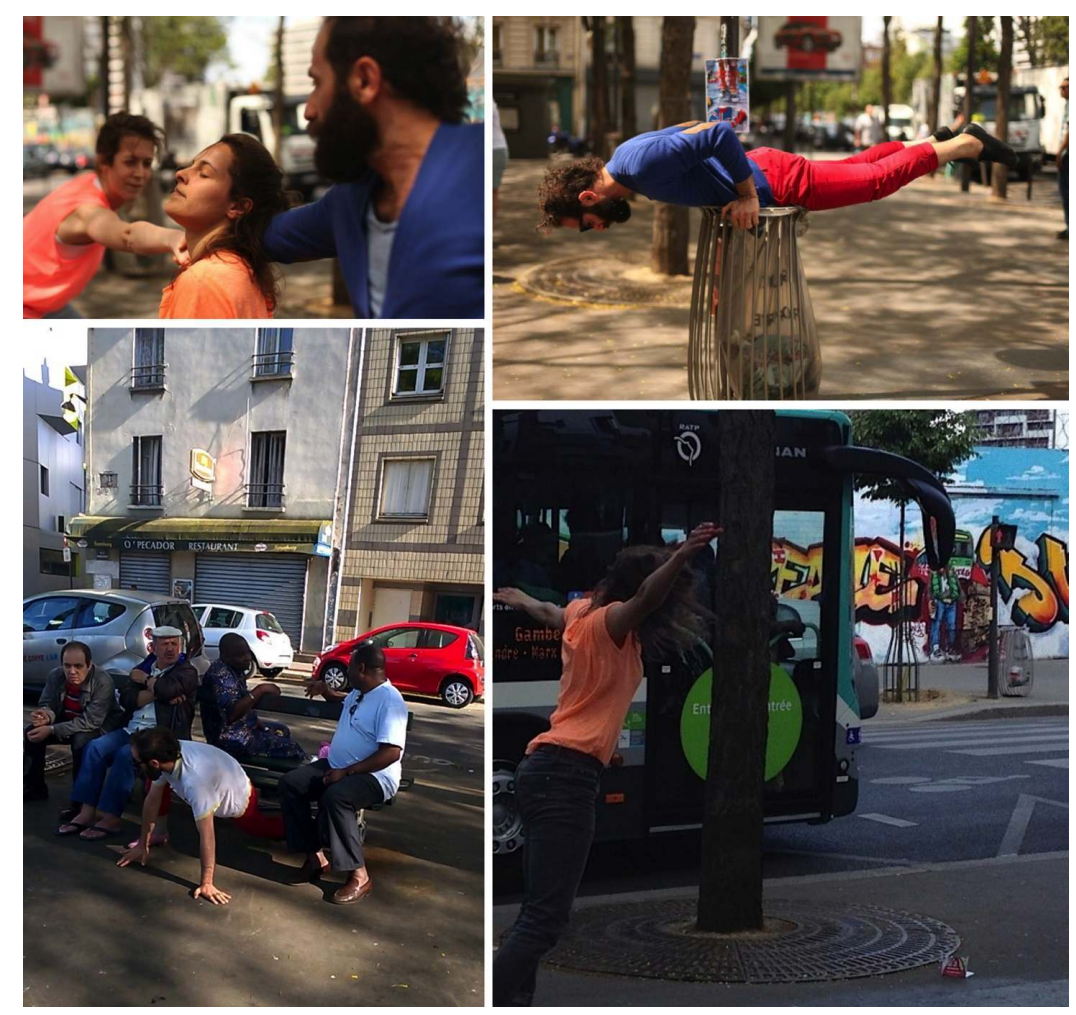

Source et copyright : Archive personnelle. @ Léna Massiani

\section{Perspectives}

La posture que j'ai adoptée au travers du premier temps fort de Danse, la cité, et qui s'est précisée au cours du deuxième n'est pas celle d'une collaboration au sens premier du terme. Avec les habitants, Danse, la cité s'est en effet organisée comme un atelier chorégraphique. A partir du sujet traité, ils ont été invités à découvrir un site selon des axes de lecture prédéfinis. Orienter la découverte $a$, semble-t-il, permis à chacun d'entrer dans l'espace avec une plus grande liberté. En s'appropriant les consignes, c'est une multitude d'approches qui donne à découvrir le Mail de l'îlot Bièvre et la place Louis Baillot. Le terme qualifiant la relation qui s'est développée avec les habitants serait sans doute davantage de l'ordre du partage. Et plus précisément, ce sont les expériences des 
uns et des autres qui ont été mises à l'écoute, celles quotidiennes en regard de celles vécues au travers de la découverte sensible des places. De là, les discussions ont non seulement fait émerger des thèmes chorégraphiques à partir desquels écrire la danse, mais également une histoire, un récit. A partir de l'expérience corporelle chacun a été en mesure de raconter son histoire qui, mise en partage, a fait émerger un récit collectif. Ainsi, à partir d'une même approche (notre relation aux places publiques) les deux temps forts de Danse, la cité racontent des histoires différentes.

A l'intérieur de ces histoires, il fallait permettre aux habitants une gestuelle faisant état de leur relation particulière avec le Mail de l'îlot Bièvre ou la place Louis Baillot. A l'endroit de l'écriture chorégraphique, j'ai donc pris le parti de proposer des directives comme des sous-thèmes à explorer et à tester. L'objectif étant que les habitants puissent poser et déposer un geste en adéquation avec l'histoire qu'ils souhaitaient raconter. Tout en échangeant avec les participants, mon rôle a été celui de mettre en relation les différentes étapes des récits, de les scénographier. De la découverte des sites à la présentation en public, nous avions chacun des tâches différentes à conduire. Faisant tous partie du même projet collectif, ensemble nous nous sommes investis dans l'élaboration de la performance dansée.

41 Si les histoires que racontent les deux performances sont différentes, le processus permettant de les faire émerger est identique, voire méthodique. Avec ce projet, afin d'optimiser la rencontre du corps sensible des habitants avec le Mail de l'îlot Bièvre ou la place Louis Baillot, il me semblait important de suivre un certain ordre dans l'approche des sites par le corps. Et le chorégraphique permet cela : les habitants ont commencé par une découverte des espaces internes de leur corps pour ensuite ouvrir et s'ouvrir à la conscience kinesthésique de celui-ci et enfin se projeter et agir avec et dans l'environnement dans lequel ils se trouvaient.

Le protocole de lecture sensible de l'espace urbain qui a émergé dans le cadre de Danse, la cité retient désormais toute mon attention pour appréhender, sous l'angle du sensible, les espaces publics que nous traversons et dans lesquels nous vivons. En choisissant les destinataires et les cadres d'utilisation, il s'agit de comprendre, de ce point de vue là, l'urbanité en fabrication.

\section{BIBLIOGRAPHIE}

Amagatsu, Ushio. 2000. Dialogue avec la gravité. Paris : Actes Sud.

Ardenne, Paul. 2002. Un art contextuel. Création artistique en milieu urbain, en situation, d'intervention, de participation. Paris : Flammarion.

Augoyard, Jean-François. 2000. L'action artistique dans l'espace urbain. In : Métral, J. (eds.).

Cultures en ville, où de l'art et du citadin. La Tour d'Aigues : Editions de l'Aube. p. 17-33.

Augoyard, Jean-François. 2005. L'expérience esthétique ordinaire de l'architecture-parcours en espace public. In : Bajolet, Emilie ; Mattéi, Marie-Flore, Jean-Marc, Rennes (eds.). Quatre ans de recherches urbaines 2001-2004. Tours : Presse universitaire François Rabelais. p. 35-40. 
Bernard, Michel. 1995. Le corps. Paris : Le seuil.

Bernard, Michel. 2001. De la création chorégraphique. Paris Pantin : Centre national de la danse.

Bruneau, Monik ; Villeneuve, André (eds.). 2007. Traité de recherche-création en Art. Québec :

Presse Universitaire du Québec.

Fraser, Marie. 1999. Sur l'expérience de la ville. In : Fraser, Marie ; Gougeon, Diane ; Perrault, Marie (eds.). Sur l'expérience de la ville : interventions en milieu urbain. Montréal : Optica, un centre d'art contemporain. p. 14-41.

Godard, Hubert. 1998. Le geste et sa perception. In : Michel, Marcelle ; Ginot, Isabelle (eds.). La danse au XX $X^{e}$ siècle. Paris : Larousse-Bordas.

Gosselin, Pierre ; Le Coguiec, Éric (eds.). 2006. La recherche-création, pour une compréhension de la recherche en pratique artistique. Québec : Presse Universitaire du Québec.

Jacob, Louis. 2007. L'art au détour de la société : les pratiques artistiques participatives. In : Culture pour tous : 10 ans des journées de la culture. Québec, Trois-Rivières : Editons d'art Le Sabord. p. 32-35.

Laban, Rudolf. 1994. La maîtrise du mouvement. Paris : Actes sud.

Louppe, Laurence. 2000. La poétique de la danse contemporaine. Bruxelles : Contredanse.

Massiani, Léna. 2011. Danse in situ, la relation danseurs, public, site. Thèse de doctorat. Montréal : Université du Québec à Montréal.

Passeron, René. 1996. La naissance d'Icare. Éléments de poḯtique générale, Saint-Germain-en-Laye et Valenciennes : Ae2cg Éditions.

\section{NOTES}

1. La performance a été présentée au jury de thèse lors de Nuit blanche Montréal (février 2010). Elle a ensuite été programmée dans le cadre du festival offta (mai 2010, Montréal), puis lors de Nuit blanche Paris (le 6 octobre 2012).

2. Une recherche en pratique artistique peut prendre plusieurs formes. Celle de la recherchecréation consiste à s'engager dans une recherche à partir de la pratique. Il s'agit ensuite de réaliser dans une même visée un geste artistique (une création) et la production d'un discours.

3. La poḯtique, science de l'art en train de faire s'adaptant au cadre de la recherche-création et à la manière dont le chercheur se trouve au cœur de l'objet qu'il étudie, se fait alors autopoḯtique.

4. Le projet s'est déroulé du 14 au 25 avril 2014, au centre d'animation Daviel dans le $13^{\mathrm{e}}$ arrondissement de Paris.

5. Exposition présentée au centre d'animation Daviel du 6 au 31 janvier 2014.

6. Rafik Slama, Mathilde Gaudin, Clémence Brodin.

7. Anne Guillemin et Mathilde Vrignaud.

8. Le projet s'est déroulé du 23 mai au 13 juin 2015, avec l'association Ab Ovo dans le $18^{\text {e }}$ arrondissement de Paris.

9. Irène Panzani et Juan Golan.

10. M. Vrignaud (le nombre de danseuses varie selon les budgets qui sont alloués au projet).

11. Selon Laban (1994), la kinésphère est le volume sphérique imaginaire qui entoure le corps, et dont l'étendue se limite à la portée maximale des membres, sans que la personne ne change de place. Le corps se trouve au centre de celle-ci. Ce terme est habituellement utilisé afin de 
démarquer l'espace individuel du mouvement, de l'espace général alentour dans lequel l'action et le déplacement prennent place. Laban a modélisé la kinésphère dans l'icosaèdre : celle-ci se structure en reliant entre eux les coins de chacun des trois plans qui, selon le théoricien, orientent le corps dans l'espace : le plan sagittal, horizontal, vertical. Sa limite est encore une fois définie par la portée des membres s'engageant dans chacun des coins de ces trois plans, dans la hauteur, la largeur, et la profondeur.

12. Dans le cadre de cet article, je propose un récit des discussions et des échanges entre les participants : impressions communes et façon d'entrer dans les sites.

\section{RÉSUMÉS}

Danse, la cité est une action artistique urbaine qui interroge la relation entre les habitants d'un quartier et une place publique. C'est donc en leur présence qu'elle s'élabore. Ceux-ci sont invités à observer leur environnement quotidiennement traversé, ils sont invités à l'éprouver par un geste expérimental, pour enfin l'investir grâce à une performance dansée. La recherche qui a été mise en œuvre avec les habitants est issue de l'observation sensible que tous les participants ont réalisé au préalable. Celle-ci utilise les caractéristiques du chorégraphique comme un moyen d'identifier l'effet d'un espace sur le corps. En filigrane, le projet ouvre la voie d'une démarche singulière : lire et comprendre l'espace urbain par le corps sensible.

Danse, la cité is an artistic urban act which aims to highlight the relationship between the inhabitants of a neighbourhood and a public area. They are invited to look at their environment, feel it with experimental gestures, in order to move in this place thanks to a site-specific dance performance. The process used with the inhabitants comes from sensory observation, previously undertaken by the whole team, which takes the choreographic characteristics as a way to identify the side effects of an area on the body. This project opens a path to reading and understanding the urban space through the sensing body.

\section{INDEX}

Keywords : Site specific dance performance, body, sensitive knowledge, urban space, public area, experimentation, performance art in urban space, participatory performance art

Mots-clés : Danse in situ, corps, connaissance sensible, espace urbain, place publique, expérimentation, action artistique urbaine, pratique artistique participative

\section{AUTEUR}

\section{LÉNA MASSIAN}

Le rapport qu'entretiennent la danse et l'espace urbain nourrit la recherche chorégraphique de Léna Massiani. Titulaire d'un Doctorat en Études et Pratiques des Arts (UQAM, 2012), elle a enseigné à l'ESA, à l'ENSAL et est chercheure associée au Centre d'Etude des Arts Contemporains de Lille 3 où elle prend part au groupe de recherche méthodologique, Dialogue entre Arts et 
Recherche (DeAR). Depuis novembre 2015, elle est également chercheure associée au Cresson. La recherche-création qu'elle met en œuvre pose les principes du chorégraphique comme la base sur laquelle développer un outil permettant une lecture de l'espace urbain par le corps sensible. lenamassiani@hotmail.com 